\title{
Pontos de Névoa e Fluidez em Biodiesel Produzido por Misturas de Lipídios
}

Letícia Thaís Chendynski², Karina Gomes Angilleli ${ }^{3}$ Bruna Aparecisa Denobi Ferreira ${ }^{4}$, Cézar Henrique Ferreira Rodrigues ${ }^{4}$, Dionisio Borsato ${ }^{5}$

${ }^{1}$ Aceito para publicação no $3^{\circ}$ Trimestre de 2014

2 Mestranda na Universidade Estadual de Londrina- UEL, leticiachendynski@gmail.com

${ }^{3}$ Doutoranda na Universidade Estadual de Londrina- UEL, kaangilelli@ yahoo.com.br

4 Bolsistas de Iniciação Científica na Universidade Estadual de Londrina- UEL, brudenobi@gmail.com, cazarhfrodrigues@ hotmail.com

${ }^{5}$ Docente na Universidade Estadual de Londrina- UEL, dborsato@uel.com

\section{Resumo}

O presente trabalho avaliou os pontos de névoa e fluidez de biodiesel obtidos pela reação de transesterificação de misturas de óleo de soja, gordura bovina e de ave, utilizando hidróxido de sódio como catalisador inorgânico na rota metílica. A análise de variância indicou que os modelos propostos podem ser utilizados para fins preditivos, pois foram significativos com $\mathrm{p}=0,008$ e $\mathrm{R}^{2}=1,0000$ para o Ponto de Névoa e com $\mathrm{p}=0,0003$ e $\mathrm{R}^{2}=0,9877$ para o Ponto de Fluidez. Além disso, para ambos os casos estudados, o desvio de regressão se mostrou não significativo. $\mathrm{O}$ valor ótimo pode ser obtido quando se utiliza 75,0\% de óleo de soja, 20,0\% de gordura de ave e 5,0\% de gordura bovina. A modelagem utilizando o delineamento experimental de mistura simplex centroide mostrou ser eficiente e relativamente simples como estratégia de otimização podendo ser considerado de grande utilidade na pesquisa e desenvolvimento de biocombustíveis.

\section{Palavras-chave}

Delineamento experimental, mistura ternária, pontos de fluidez e de névoa 


\begin{abstract}
The present study evaluated pour and cloud points of biodiesel produced by transesterification reaction of mixtures of crude soybean oil, beef tallow and poultry fat, using sodium hydroxide as catalyst in inorganic methyl route. The analysis of variance indicated that the proposed model can be used for predictive purposes, as were significant with $\mathrm{p}=$ 0.0021 and $R^{2}=0,9911$ for the Cloud Point and $p=0.0023$ and $R^{2}=0.9903$ for the Pour Point. Moreover, in both cases studied, the deviation regression showed insignificant. The optimum value was obtained when using $75.0 \%$ soybean oil, $5.0 \%$ beef tallow and $20,0 \%$ poultry fat. The modeling using the response surface methodology is efficient and relatively simple as optimization strategy can be considered very useful in the research and development of biofuels.
\end{abstract}

\title{
Keywords
}

Experimental design, ternary mixture, cloud and pour point

\section{Introdução}

Durante décadas desde a descoberta dos combustíveis fósseis, o petróleo tem sido a principal matéria prima, porém este não é um recurso renovável, além de ser extremamente poluente. A fim de fornecer energia de maneira sustentável, renovável e degradável, o biodiesel tem se destacado como uma fonte energética alternativa não tóxica (BORSATO et al., 2012; CINI et al., 2013; SIMÕES, 2013; HAYYAN, 2013).

O biodiesel é um exemplo prático do emprego da biomassa para produção de energia, sendo que este apresenta diversas vantagens sobre o diesel de petróleo como baixa/mínima toxidade e ser proveniente de fontes renováveis (BORSATO et al., 2012 e PEREIRA, 2012).

Além das vantagens relacionadas a fatores ambientais, ecológicos e econômicos (NURTIFRI, 2013 e CANESIN, 2014), é necessário citar as melhorias nas propriedades combustíveis tais como a ausência de enxofre e compostos aromáticos, não havendo formação de dióxido de enxofre; número de cetano elevado, portanto, elevado poder de autoignição e 
combustão; maior ponto de fulgor, que fornece uma maior segurança pela baixa volatilidade, apesar de reduzir a lubrificação do motor (CANDEIA, 2008).

A queima do biodiesel gera dióxido de carbono, um dos gases responsável pelo efeito estufa, porém o mesmo é consumido pelas plantas oleaginosas utilizadas para a extração do óleo utilizado na síntese. Portanto, há uma diminuição dos impactos ambientais referentes aos subprodutos gerados a partir da combustão deste combustível, sendo que para ser obtido um ciclo fechado é necessário utilizar a rota etanolica (BORSATO et al., 2010; MAIA, 2011)

Há mais de 100 anos um inventor chamado Rudolph Diesel (1858-1913) projetou motor do ciclo Diesel para funcionar com óleos vegetais, sendo que esse era mais eficiente que os motores a vapor da época. Demonstrou esse invento abastecendo o motor com óleo de amendoim durante uma exposição parisiense (SIMÕES, 2013). Entretanto, a aplicação direta de óleos vegetais no motor pode gerar alguns danos ao mesmo, pois pode ocorrer combustões incompletas e problemas na atomização do combustível devido às características físicoquímicas desses óleos, tais como a alta viscosidade e o caráter poliinsaturado (FERRARI, OLIVEIRA, SCABIO, 2005).

Transesterificação também não é um processo recente, sendo que cientistas já utilizavam, em 1853, essa reação para fornecer combustível para veículos na África do Sul antes da $2^{\text {a }}$ Guerra Mundial (DEMIRBAS, 2003).

É importante lembrar que o Brasil se destaca no cenário mundial energético com os biocombustíveis. Na bioenergia, a cana-de-açúcar também se sobressai pelos avanços tecnológicos, na fase agrícola e na industrial com o etanol e a "bioeletricidade", para que estes sejam produtos competitivos. Com relação ao biodiesel, a maior parte produzida no Brasil é utilizada, por enquanto, para atendimento da norma de conformidade prevista por lei (TOLMASQUIM, 2012). De acordo com as propostas de sustentabilidade, pode-se produzir biocombustíveis a partir de óleos de frituras e de sebo bovino (ORIVES et al., 2014), reduzindo ainda mais os riscos de poluição ambiental causados por esses materiais quando descartados erroneamente na natureza. A alta disponibilidade e baixo preço da gordura animal está gerando interesse industrial, com a sua utilização em conjunto com o óleo de soja para a produção de biodiesel, pode diminuir custos (BORSATO et al.,2010; ORIVES et al., 2014). 
O presente trabalho objetiva estudar as características físico-químicas de biodiesel (ponto de névoa e ponto de fluidez) obtido pela reação de transesterificação de misturas de óleo de soja, gordura bovina e de ave.

\section{Materiais e métodos}

A reação de transesterificação dos triglicerídeos, sem antioxidantes, da mistura de óleo de soja refinado (Imcopa ${ }^{\circledR}$, Londrina-PR), gordura de ave (Big Frango, Rolândia-PR) e gordura bovina comercial $\mathrm{Cristal}^{\circledR}$ foi efetuada com álcool metílico PA ACS (Anidrol ${ }^{\circledR}$, 99,8\%), juntamente com catalisador inorgânico hidróxido de sódio ( $\mathrm{NaOH}, \mathrm{F}$. Maia $\left.{ }^{\circledR}, 97,0 \%\right)$, sob refluxo e aquecimento controlado a $65^{\circ} \mathrm{C}$, com auxílio de um termômetro, e agitação magnética em um balão de 3 bocas específico para síntese (Laborglas®, 250mL) acoplado em um condensador (Vidrolabor $\left.{ }^{\circledR}\right)$.

Lavou-se a referida com $100 \mathrm{~mL}$ de uma solução de ácido acético glacial 0,25\%(v/v), a $80^{\circ} \mathrm{C}$ e, em seguida, com água destilada a $80^{\circ} \mathrm{C}$ até o $\mathrm{pH}$ neutro.

O ponto de névoa e de fluidez foi realizado em um recipiente próprio para esse tipo de teste, com um termômetro externo (ASTM 5C-86), mantido a $-20^{\circ} \mathrm{C}$ por uma mistura de gelo e cloreto de sódio, e um outro termômetro (ASTM 5C-86) na amostra para mensurar a temperatura em que a amostra inicia a solidificação (ponto de névoa) e a temperatura em que a amostra deixa de fluir (ponto de fluidez), em conformidade com a norma ASTM D2500-05 (ASTM International, 2005).

Foi utilizado delineamento experimental de misturas simplex-centroide, contendo $2^{\mathrm{q}}$ 1 ensaios com uma repetição no ponto central, onde $q$ representa o número de variáveis independentes. representadas na equação por $\mathrm{x}_{1}$ (Teor de óleo de soja), $\mathrm{x}_{2}$ (Teor de Gordura bovina) e $\mathrm{x}_{3}$ (Teor de gordura de ave). As análises estatísticas foram realizadas no Statistic 9.0. As respectivas proporções do delineamento estão apresentadas na Tabela 1.

Tabela 1. Delineamento experimental de misturas simplex-centroide

\begin{tabular}{cccc}
\hline \multicolumn{5}{c}{ Proporções (\%) } \\
\hline & Óleo de soja & Gordura Bovina & Gordura de ave \\
\hline 1 & 1,00 & 0,00 & 0,00 \\
2 & 0,00 & 1,00 & 0,00 \\
3 & 0,00 & 0,00 & 1,00
\end{tabular}




\begin{tabular}{llll}
4 & 0,50 & 0,50 & 0,00 \\
5 & 0,50 & 0,00 & 0,50 \\
6 & 0,00 & 0,50 & 0,50 \\
7 & 0,33 & 0,33 & 0,33 \\
8 & 0,33 & 0,33 & 0,33 \\
\hline
\end{tabular}

\section{Resultados e discussão}

A Tabela 2 mostra o delineamento experimental utilizado e os valores obtidos para o ponto de névoa e de fluidez.

Tabela 2. Resultados do delineamento experimental de misturas simplex-centroide

\begin{tabular}{|c|c|c|}
\hline & Ponto de Névoa $\left({ }^{\circ} \mathrm{C}\right)$ & Ponto de Fluidez $\left({ }^{\circ} \mathrm{C}\right)$ \\
\hline 1 & 3,00 & $-2,00$ \\
\hline 2 & 22,00 & 14,00 \\
\hline 3 & 9,00 & 3,00 \\
\hline 4 & 15,00 & 8,00 \\
\hline 5 & 5,00 & 0,00 \\
\hline 6 & 13,00 & 9,00 \\
\hline 7 & 9,00 & 7,20 \\
\hline 8 & 9,10 & 7,00 \\
\hline
\end{tabular}

Com a aplicação do delineamento experimental de mistura simplex-centroide, foram obtidos os modelos a seguir, contendo apenas os termos significativos em nível de 5\%, representados pelas equações 1 e 2 para as respostas das variáveis dependentes do Ponto de Fluidez $\left(\mathrm{Y}_{1}\right)$ e do Ponto de Névoa $\left(\mathrm{Y}_{2}\right)$, respectivamente.

$$
\begin{gathered}
Y_{1}=-2,13 x_{1}+14,27 x_{2}+3,32 x_{3}+10,47 x_{1} x_{2}(1) \\
Y_{2}=3,00 x_{1}+22,00 x_{2}+9,00 x_{3}-10,00 x_{1} x_{2}-4,00 x_{1} x_{3}-10 x_{2} x_{3}-49,65 x_{1} x_{2} x_{3}
\end{gathered}
$$

Onde $\mathrm{x}_{1}$ representa a proporção de óleo de soja, $\mathrm{x}_{2}$, de gordura bovina, e $\mathrm{x}_{3}$, de Revista Brasileira de Energias Renováveis, v. 3, p.307- 316, 2014 
gordura de ave.

As análises de variância (Tabela 3 e 4) indicaram que os modelos propostos podem ser utilizados para fins preditivos, pois foram significativos com $\mathrm{p}=0,03$ e $\mathrm{R}^{2}=1,0000$ para o Ponto de Névoa e $\mathrm{p}=0,0003$ e $\mathrm{R}^{2}=0,9877$ para o Ponto de Fluidez. Além disso, para ambos os casos estudados, o desvio de regressão se mostrou não significativo.

Tabela 3. Análise de variância para a resposta ponto de névoa $\left({ }^{\circ} \mathrm{C}\right)$

\begin{tabular}{llllll}
\hline Fonte de variação & G.L. & Soma dos quadrados & Quadrado médio & F & p \\
\hline Modelo & 5 & 248,56 & 49,71 & 33,16 & 0,03 \\
Erro total & 2 & 3,00 & 1,50 & & \\
Total ajustado & 7 & 251,56 & 35,94 & & \\
\hline
\end{tabular}

Tabela 4. Análise de variância da resposta ponto de fluidez $\left({ }^{\circ} \mathrm{C}\right)$

\begin{tabular}{llllll}
\hline Fonte de variação & G.L. & Soma dos quadrados & Quadrado médio & F & p \\
\hline Modelo & 3 & 185,73 & 61,91 & 107,29 & 0,0003 \\
Erro total & 4 & 2,31 & 0,58 & & \\
Total ajustado & 7 & 188,04 & 26,86 & & \\
\hline
\end{tabular}

A região de combinação ternária entre as variáveis originais (gordura de ave, gordura bovina e óleo de soja) pode ser observada por meio das curvas de nível apresentadas na Figura 1. A figura obtida mostra as regiões de contorno para a variável dependente, ponto de névoa e fluidez, obtidas pelo modelo matemático.

Figura 1. Superfície de resposta para (a) Ponto de Fluidez $\left({ }^{\circ} \mathrm{C}\right)$ e para (b) Ponto de Névoa $\left({ }^{\circ} \mathrm{C}\right)$ a partir do delineamento experimental proposto 
(a)

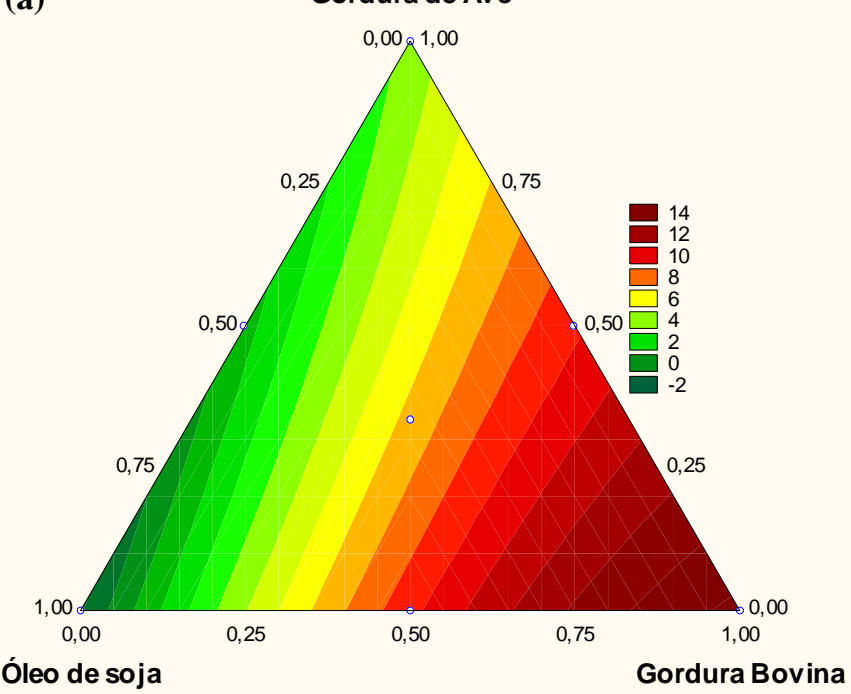

(b)

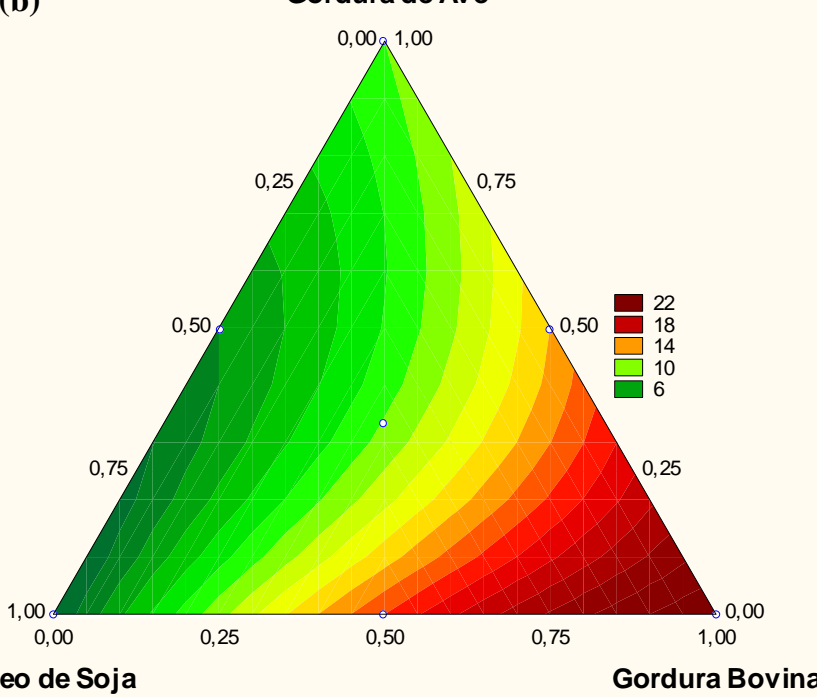

Com base na Figuras 1a e 1b observa-se que a região ótima, para o ponto de névoa e fluidez, situa-se próxima ao ponto central do delineamento experimental.

O gráfico de Pareto contendo apenas os efeitos significativos (Figuras 2a e 2b), em nível de $95 \%$ de confiança, mostra a ordem de importância das variáveis empregadas no modelo preditivo obtido. Os valores ao lado do retângulo representam os valores da estatística do teste t.

FIGURA 2. (a) Gráfico de Pareto para Ponto de Fluidez $\left({ }^{\circ} \mathrm{C}\right)$ e (b) para o Ponto de Névoa $\left({ }^{\circ} \mathrm{C}\right)$ a partir do delineamento experimental proposto

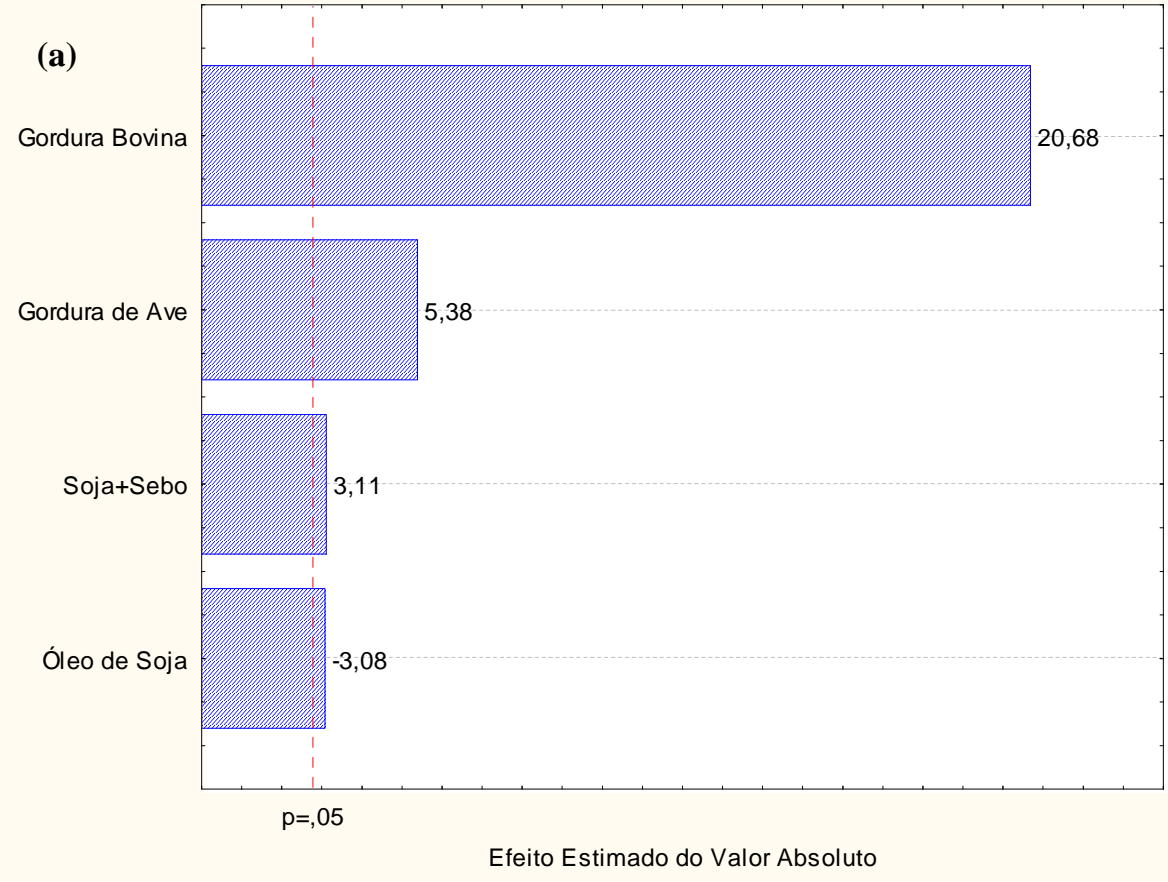




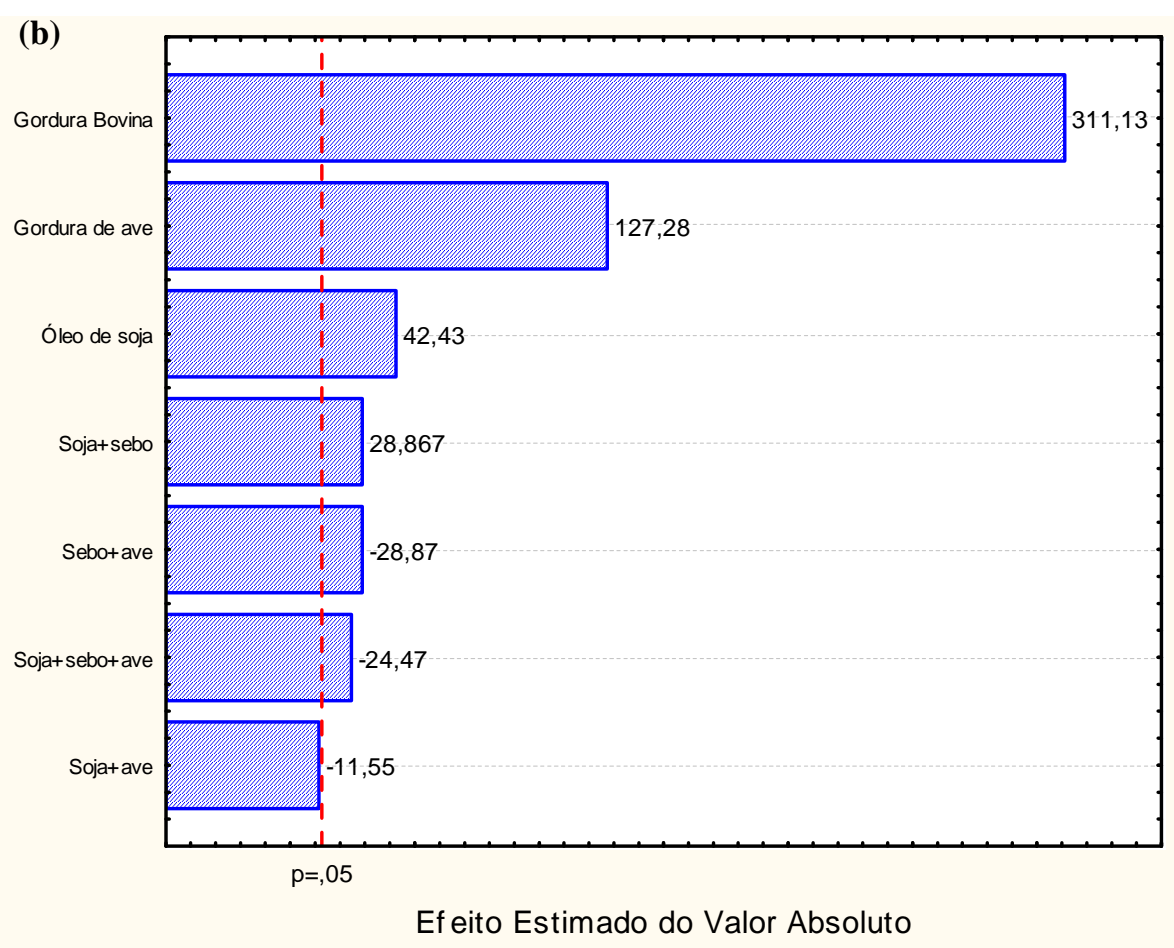

A Figura 3 mostra a otimização multirresposta das variáveis empregando o software Statistica 9.0. Foi estabelecido o valor alvo de $4{ }^{\circ} \mathrm{C}$ para o ponto de nevoa e $0{ }^{\circ} \mathrm{C}$ para o ponto de fluidez. A figura mostra que o valor ótimo pode ser obtido quando se utiliza 75,0 \% de óleo de soja, $20,0 \%$ de gordura de ave e $0,5 \%$ de gordura bovina na produção do biodiesel sob as condições descritas.

FIGURA 3. Otimização conjunta multirresposta do ponto de Névoa e do ponto de Fluidez
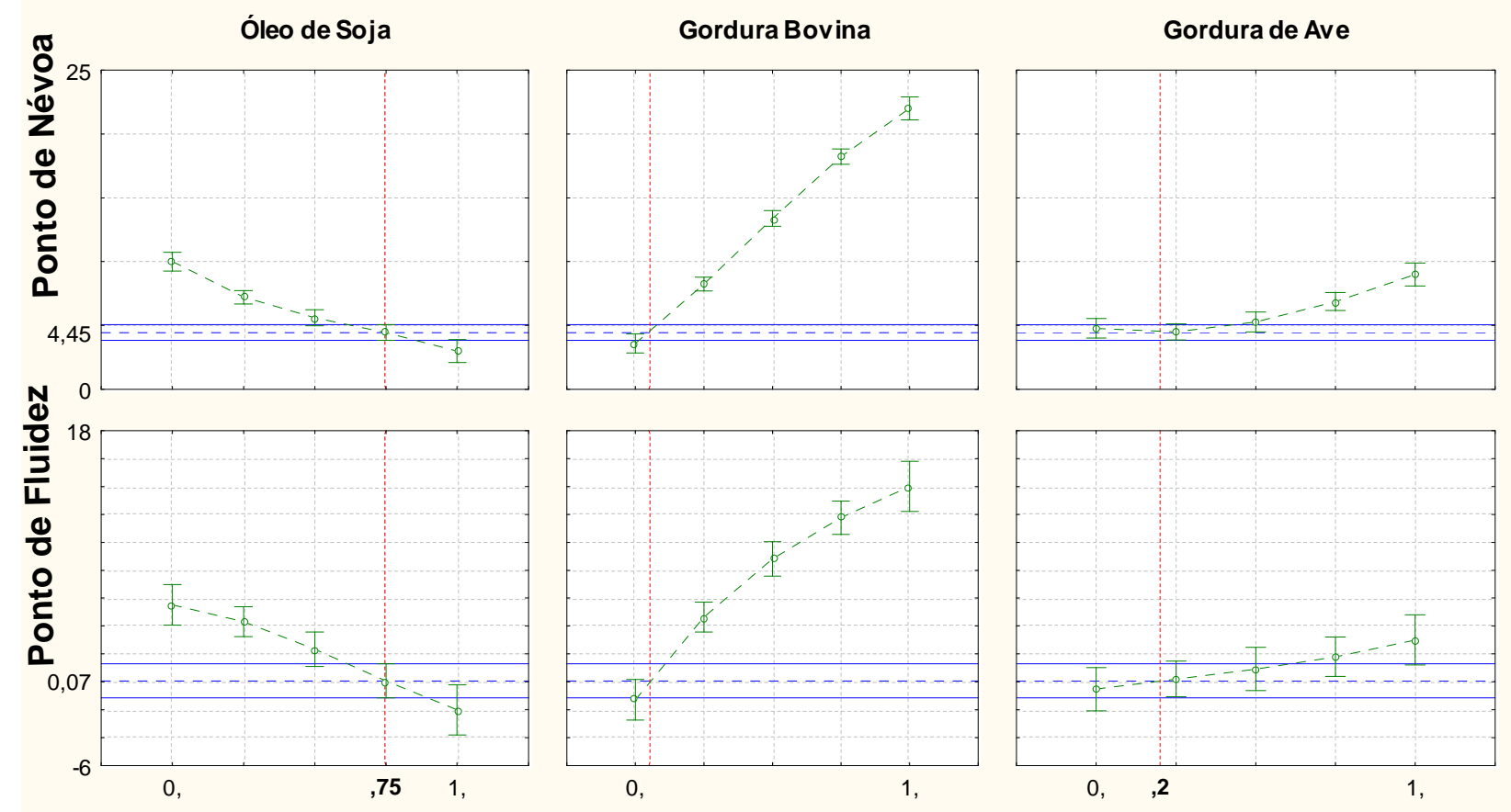

Revista Brasileira de Energias Renováveis, v. 3, p.307- 316, 2014 


\section{CONCLUSÃO}

A modelagem utilizando o delineamento experimental de misturas simplex-centroide pode ser considerada de grande utilidade na pesquisa e desenvolvimento de biocombustíveis, sendo que a partir dos gráficos obtidos, observa-se uma boa otimização e ajuste ao modelo apresentado.

\section{AGRADECIMENTOS}

À CAPES, CNPQ e Fundação Araucária pela concessão de bolsas de estudos como auxílio financeiro, à UEL e ao Laboratório de Pesquisa e Análise de Combustíveis pela infraestrutura e equipamentos concedidos.

\section{REFERÊNCIAS BIBLIOGRÁFICAS.}

ASTM International; ASTM D2500-05 - Standard Test Method for Cloud Point of Petroleum Products, 2005.

BORSATO, D.; MAIA, E. C. R.; DALl'ANTONIA, L. H.; SILVA, H. C.; PEREIRA, J. L. Cinética da oxidação do biodiesel de óleo de soja em mistura com TBHQ: Determinação do tempo de estocagem. Química Nova. v.35, n.4, p.733-737, 2012.

BORSATO, D.; DALL'ANTONIA, L. H.; GUEDES, C. L.; MAIA, E. C. R.; FREITAS, H. R.; MOREIRA, I.; SPACINO, K. R. Aplicação do delineamento simplex-centroide no estudo da cinética de oxidação de biodiesel B100 em mistura com antioxidantes. Química Nova, v. 33, n. 8, p. 1726-1731,2010.

CANDEIA, R. A. Biodiesel de soja: Síntese, degradação e mistura binárias.Tese.Universidade Federal da Paraíba. 2008.150p.

CANESIN, E. A.; OLIVEIRA, C. C.; MATSUSHITA, M.; DIAS, L. F.; PEDRÃO, M. R. SOUZA, N. E. Characterization of residual oils for biodiesel producion. Electronic Journal of Biotechnology. v. 17, p.39-45, 2014.

CINI, J. R. M.; BORSATO, D.; GUEDES, C. L. B.; SILVA, H. C.; COPPO, R. L. Comparação dos métodos de determinação estabilidade oxidativa do biodiesel B100 em mistura com antioxidantes sintéticos. Aplicação do delineamento simplex-centroide com variável de processo. Química Nova. v. 36, n.1, p.79-84, 2013.

FERRARI, R. A.; OLIVEIRA, V. S.; SCABIO, A. BIODIESEL DE SOJA - Taxa de conversão em ésteres etílicos, caracterização físico-química e consumo em gerador de 
energia. Química Nova. v. 28, n. 1, p. 19-23, 2005.

HAYYAN, A.; HASHIM, M. A.; MJALLI, F. S.; HAYYAN, M.; ALNASHEF, I. M. A novel phosphonium-based deep eutectic catalyst for biodiesel production from industrial low grade crude palm oil. Chemical Engineering Science, v. 92, p. 81-88, 2013.

MAIA, E. C. R. Estudo da oxidação do biodiesel B100 em mistura com antioxidantes. Aplicação do delineamento simplex-centroide. 2011.96f. Dissertação (Mestrado em Química). Universidade Estadual de Londrina, Londrina.

DEMIRBAS, A. Biodiesel fuel from vegetables oils via catalytic and non-catalytic supercritical alcohol transesterification and other methods: a survey. Energy Conversion and Management, v. 44, p.2093-2109, 2003.

NURTIFRI, I.; MANIAM, G. P.; HINDRYAWATI, N; YUSOFF, M. M.; GANESAN, S. Potencial of feedstock and catalysts from waste in biodiesel preparation: A review. Energy conversion and Management. v. 74, p. 395-402, 2013.

ORIVES, J. R.; GALVAN, D.; COPPO, R. L.; RODRIGUES, C. H. F. , ANGIELLI, K. G., BORSATO, D. Multiresponse optimisation on biodiesel, obtained, through, a ternary mixture of vegetable oil and animal fat: Simplex-centroide mixture design application. Energy Conversion and Management, p.398-404, 2014.

PEREIRA, C. M. P.; HOBUSS, C. B.; MACIEL, J. V.; FERREIRA, R. L.; DEL PINO, F. B.; MESKO, M. F.; JACOB-LOPEZ, E.; NETO, P. C. Biodiesel Renovável derivado de microalgas avanços e perspectivas tecnológicas. Química Nova, v. 35, n. 10, p. 2013-2018, 2012.

SIMÕES, D. M. B. Viabilidade de utilização de mafurra (Trichilia emética) para a produção do biodiesel. 2013. Universidade de Lisboa.

TOLMASQUIM, M. T. Perspectivas e planejamento do setor energético do Brasil. Estudos Avançados. v. 26, n. 74, 2012. 